\title{
The Effects of Temperature and RF Power Level on the Tuning of the Water-Cooled SSC Low-Energy Booster Cavity
}

\author{
C. Friedrichs \\ Los Alamos National Laboratory \\ G. Hulsey \\ Superconducting Super Collider Laboratory
}

\section{Abstract}

The SSC Low-Energy Booster (LEB) cavity must rapidly tune from 47.52 to $59.78 \mathrm{MHz}$. The cavity tuner will use transversely biased ferrite[1] to control the cavity resonance.

The thermal expansion of a cavity's materials affects its resonance. There are two other known temperature mechanisms that affect resonance in the water-cooled LEB cavity. The saturation magnetization of the ferrite is a function of temperature, and since the ferrite permeability is dependent on the saturation magnetization, the ferrite permeability is also temperature dependent. The ferrite cooling water is present in the tuner rf field, hence the water permittivity, which is very temperature dependent, also affects cavity resonance.

While taking data on the SSC Test Cavity to quantify the effect of temperature on the resonance, we observed that the if power level also perturbed the resonance. It was readily apparent from the data that the power level affected the resonance much more strongly at low values of control bias than at high values. In fact, when we calculate an apparent modified control-bias $\mathrm{H}$ field that produces the observed resonance shift, we noticed an almost perfect, though non-linear, correlation between the ratio of $H_{r f}$ to $H_{\text {bias }}$ and the apparent modified bias field, $H_{a p p}$.

This paper will present a set of equations to predict the resonance shifts produced by changes in temperature and if power level. It will also present the techniques, both theoretical and empirical, by which these equations are derived. Finally, some of the methods for dealing with these resonance shifts will be discussed.

\section{INTRODUCTION}

We will consider three separate effects on the cavity tuning: the effects of temperature on ferrite permeability and on cooling water permittivity, as well as the effects of rf the power level on the ferrite permeability. These are distinctly separate effects and we will treat them separately.

We derived the effect of temperature on ferrite permeability from the fact that the rf permeability of the ferrite is a function of the saturation magnetization of the ferrite, which is in turn a function of temperature. The saturation magnetization vs. temperature is usually available from the ferrite manufacturer.

We could locate no data for the effect of temperature on the water permittivity, so measurements were made at Los Alamos National Laboratory and the Superconducting Super

\footnotetext{
"Work supported and funded by U. S. Department of Energy office at the Superconducting Supercollider Laboratory.
}

Collider (SSC) to determine the permittivity and Q of both distilled and de-ionized water in the frequency range of interest.

We do not have a theory to predict the effect of $\mathrm{rf}$ power level on cavity tuning. We have empirically derived the predictions so that they fit our measured data. We took the data using the SSC Test Cavity. We used low-duty cycles while taking this data in order to avoid mixing temperature effects with power-level effects. We recorded the frequency and frequency shift vs. If cavity-gap voltage for gap voltages from $1 \mathrm{kV}$ to $100 \mathrm{kV}$ at various ferrite bias current levels from 105 to 240 amperes. We used the data to calculate $H_{f f}$ and $H_{\text {bias }}$ in the ferrite, then used these quantities to produce a consistent empirical formula that matches the measured frequency shift.

\section{TEMPERATURE EFFECT ON PERMEABILITY}

Saturation magnetization data for Trans Tech G-810 ferrite is shown in Fig. 1.

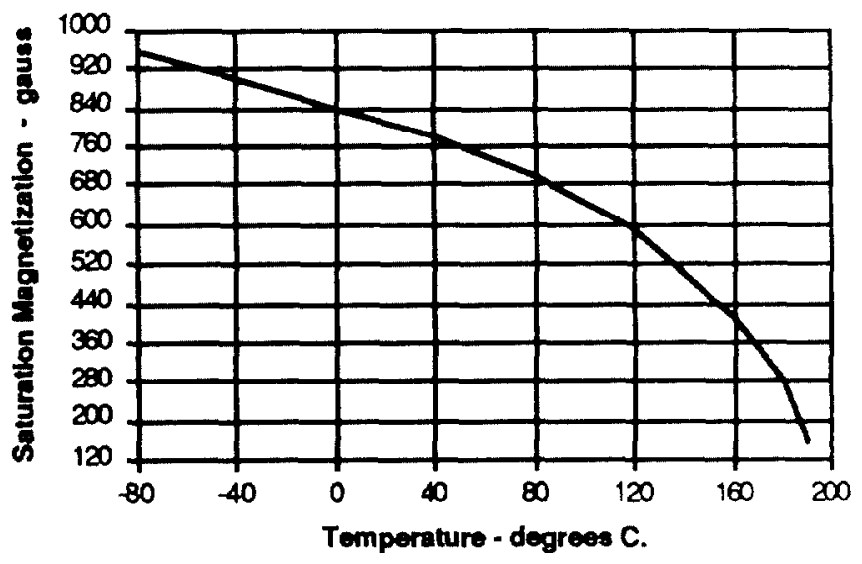

Figure 1. Saturation magnetization vs. temperature

We used curve-fitting techniques to derive a function that matches the curve. We then substituted this function into the equation defining if permeability. These expressions are given below:

$$
\begin{aligned}
& B_{s a t}=732.45 \times \log _{10}(215-T)-860.17 \\
& \mu=\frac{B_{\text {sat }}}{H_{\text {ftr }}}+1=\frac{732.45 \times \log _{10}(215-T)-860.17}{H_{\text {fer }}}
\end{aligned}
$$


The spacing between the pole pieces of the tuning magnet in the LEB water cooled cavity is $16.9 \mathrm{~cm}$. This spacing contains $12.5 \mathrm{~cm}$. of ferrite. The relation of $\mathrm{H}$ to the tuning current is given by the following expression:

$$
n \times I=\oint_{H} H \cdot d l \approx \sum H \times l \approx H_{\text {jer }} \times l_{\text {jer }}+H_{\text {air }} \times l_{\text {air }},
$$

where $I$ is the tuner bias current and $n$ is the number of turns in the bias magnet coil. Now

and

$$
H_{f e r}=B_{\text {fer }}-B_{\text {sait }}, \quad H_{\text {air }}=B_{\text {air }} / \mu_{\text {air }}
$$

$$
l_{\text {fer }}=12.5 \mathrm{~cm}, l_{\text {air }}=4.4 \mathrm{~cm} .
$$

Since $B$ is continuous, $B=B_{f e r}=B_{\text {air }}$. So

$$
B=\frac{12.5 \times B_{\text {sas }}+n I}{12.5+4.4 / \mu_{\text {air }}},
$$

and finally

$$
\mu_{\text {fer }}=\frac{B}{B-B_{\text {sat }}} \text {. }
$$

The cavity resonant frequency as a function of ferrite permeability can be calculated using either Superfish or any of the transmission line codes[2].

\section{III.TEMPERATURE EFFECT ON WATER PERMITTIVITY}

Permittivity of de-ionized water as a function of temperature is shown in Fig. 2. This data was obtained from measurements performed at $56 \mathrm{MHz}$. The permittivity is linearly decreasing with increasing temperature, and can be calculated using the following empirical expression:

$$
\varepsilon=-0.4168 \times T+86.519 \text {. }
$$

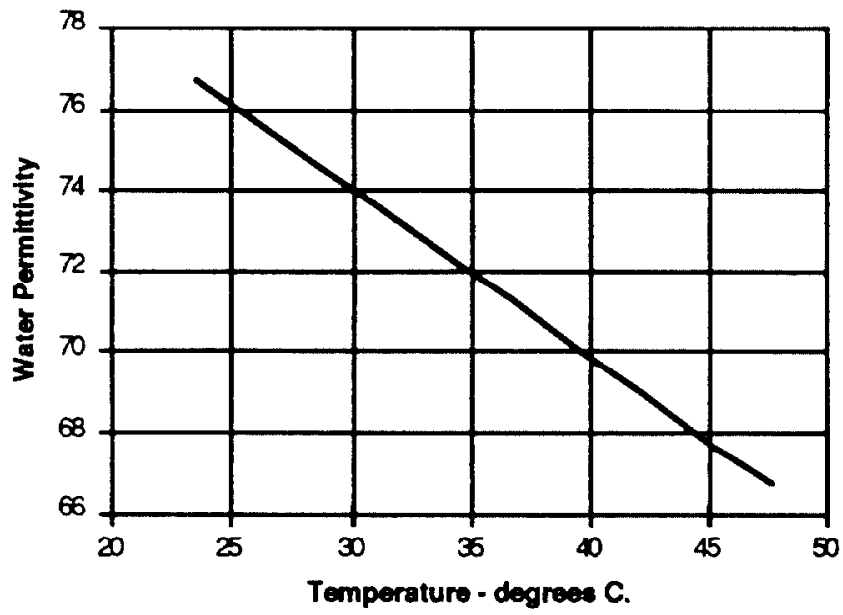

Figure 2. Permittivity vs. temperature
The cavity resonant frequency as a function of water permittivity can be calculated by the same methods mentioned in Section II.

\section{EFFECT OF POWER LEVEL ON TUNING}

We first noticed that power level had an appreciable effect on the cavity resonant frequency while we were taking data on the effects of temperature on tuning. Since the tuner and cavity we were testing were substantially different from the LEB tuner design, we felt it was important to be able to make a prediction regarding the strength of this effect in the LEB cavity. After first noticing this effect, we observed that it was much more pronounced at the lower tuning frequencies than the higher ones, or more significantly, it was much more pronounced at lower values of control bias than at higher ones.

We examined the ratio of $H_{r f}$ to $H_{\text {bias }}$ and determined that for any value of this ratio, there is a unique factor that apparently modifies $H_{\text {bias. }}$. After applying this factor to $H_{b i a s}$, a new value of ferrite permeability may be calculated using the expression for $\mu$ given in Section II of this paper. The non-linear function for the change factor that matches the measured data is shown in Fig. 3. The peak value of $H_{r f}$ is used.

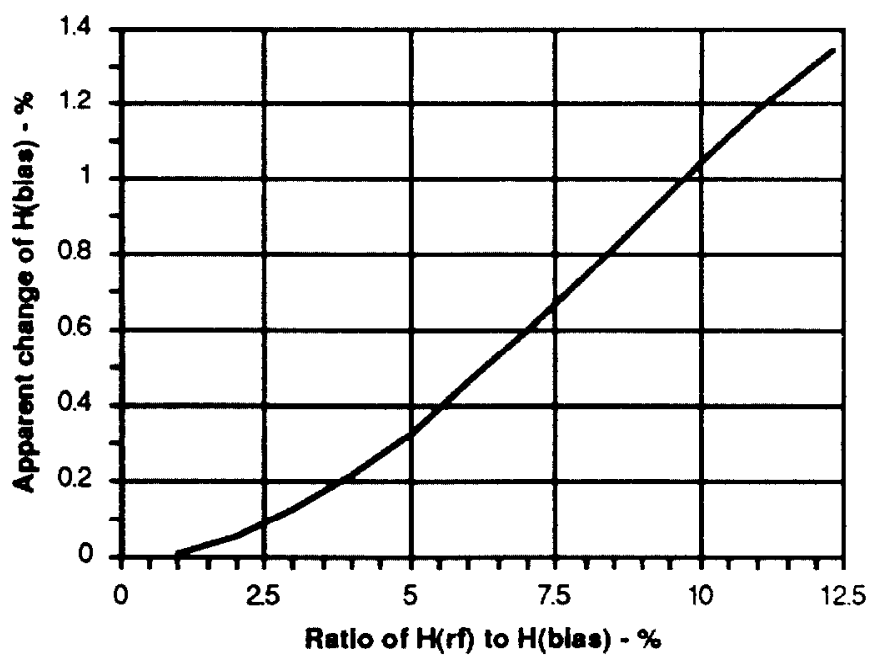

Figure 3. Effect of if amplitude on apparent $H$

Once we had quantified this change function, we next sought to realize a simple mathematical model to calculate the apparent control bias $\mathrm{H}$ field as a function of $H_{\text {bias }}$ and $H_{r f}$. The following equation expresses our first attempt at this realization ( $H_{a p p}$ is the apparent bias field):

$$
H_{\text {app }}=\sqrt{H_{r f}^{2}+H_{\text {bias }}^{2}} \text {. }
$$


This expression yields a fairly good correlation with the measured data, but its predicted frequency shift is consistently on the low side.

Rather than accept a slight error on the low side, we elected to modify the expression. $H_{a p p}$ in the above expression can be readily recognized as the vector sum of $H_{r f}$ and $H_{\text {bias }}$, with the two quantities $90^{\circ}$ apart. The simplest way to modify the expression to improve its fit to the data is to increase the angle between the two vectors. The vector addition is illustrated in Fig. 4.
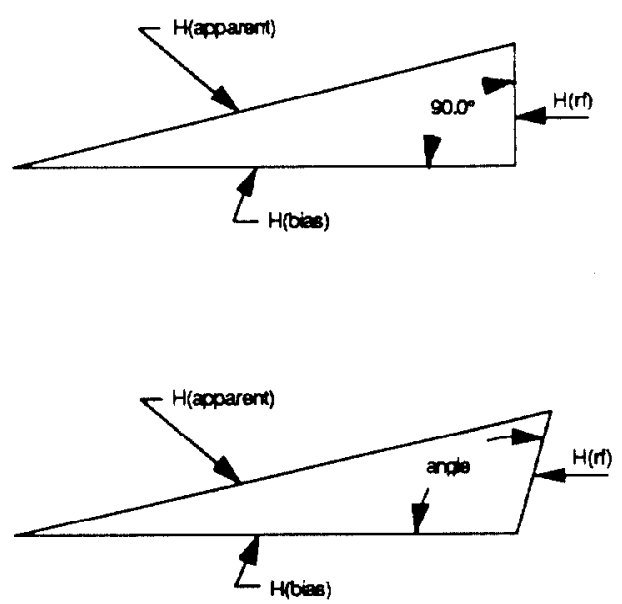

Figure 4. Construction of $H_{\text {apparent }}$

The best fit to the data occurs when the angle is increased from $90^{\circ}$ to $92.8^{\circ}$. The use of $92.8^{\circ}$ results in excellent correlation with the data, and no low side errors appear in the regions where the shift is large.

At first it seems that a frequency shift of less than $2 \%$ would not be objectionable in a circuit designed to rapidly tune over a $25 \%$ range. The problem is caused by a severe non-linearity that can result from the phenomenon. If the cavity driving frequency is swept from below resonance to above resonance, say from -6 to $+6 \mathrm{~dB}$, at an amplitude level sufficiently large to produce significant shift, the cavity impedance will avalanche just after resonance is crossed. This effect occurs because $H_{r f}$ starts decreasing causing the resonance to shift back away from the driving frequency, and a runaway condition occurs. Such an occurrence can be prevented by the application of sufficient amplitude feedback. A good prediction of the expected shift is, therefore, important to the feedback designers.

A computer simulation of the avalanche effect described above is shown in Fig. 5.

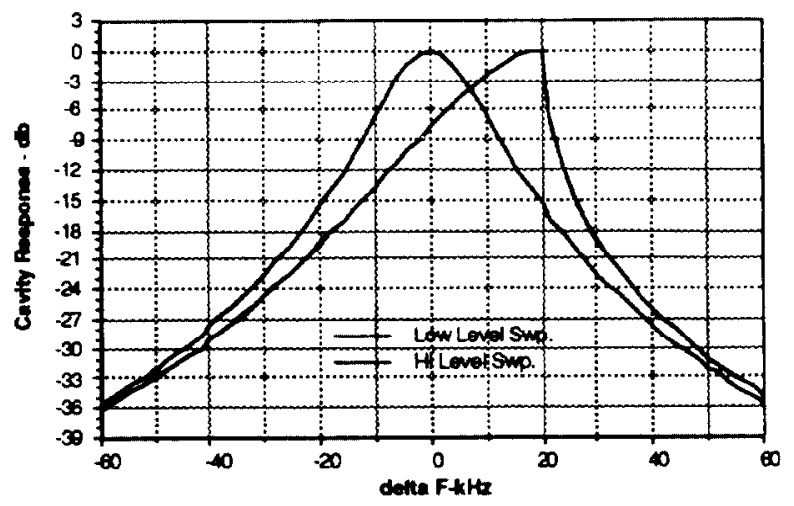

Figure 5. Avalanche effect

\section{ACKNOWLEDGMENTS}

Many people in both the if and mechanical engineering departments at SSC made significant contributions to bringing the RF Cavity Test Stand on line. We would particularly like to thank Fred Brandeberry for assistance in the measurements, and Yehuda Goren for his attempts to find a theoretical basis for the frequency shift vs amplitude phenomenon.

\section{REFERENCES}

[1] W.R. Smythe, T.G. Brophy, R.D. Carlini, C.C. Friedrichs, D.L. Grisham, G. Spalek, and L.C. Wilkerson, "RF Cavities with Transversely Biased Ferrite Tuning," IEEE Trans. Nucl. Sci. 32 (5), p. 2951. (1985).

[2] C.C. Friedrichs, "Analytic Evaluation of the LAMPF II Booster Cavity Design," IEEE Trans. Nucl. Sci. 32 (5), p. 2843 (1985). 